\title{
Steven Nissen
}

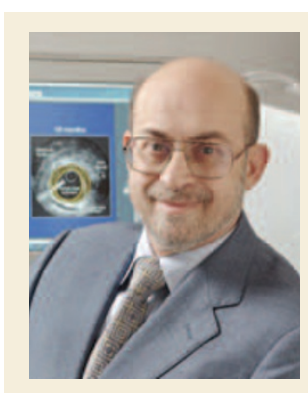

Steven Nissen, Medical Director, Cleveland Clinic Cardiovascular Coordinating Center

Steven Nissen is Medical Director of the Cleveland Clinic Cardiovascular Coordinating Center, an organization that directs multicentre clinical trials. He is a pioneer of cardiovascular imaging and his research focuses on using imaging techniques to assess the progression and regression of coronary atherosclerosis. Nissen has served as Chairman of the FDA CardioRenal Advisory Panel for 5 years and in March 2006 will begin a 1-year term as President of the American College of Cardiology. He recently took on the role of Lead Investigator of the high-profile PRECISION trial investigating the relative safety of cyclooxygenase-2 (COX2) inhibitors compared with conventional non-steroidal inflammatory drugs (NSAIDs).

\section{How did you arrive at the proposed PRECISION study design?}

We know from the VIGOR, APPROVe and APC trials that the COX inhibitors appear to increase the risk of atherothrombotic cardiovascular events including myocardial infarction (MI) and stroke compared with placebo. We've not, however, tested the conventional NSAIDS and we always assumed that they were neutral - that they didn't have the antiplatelet effects of aspirin, but that they wouldn't produce harm either. Epidemiological studies were suggesting that all of the NSAIDs posed a risk, and so we faced a fundamental conundrum in medicine: arthritis and cardiovascular risk are both extraordinarily prevalent as age increases, so the population taking anti-inflammatories overlaps substantially with people at cardiovascular risk. If a patient walks into my office with arthritis and heart disease, what do I give them? Ibuprofen, naproxen or the remaining COX inhibitor, celecoxib? The only way to answer that question is to do a massive head-to-head trial in people of sufficient risk to actually get enough cardiovascular events for precise results. That's what's driving the sample size, which currently is looking like 21,000 patients. That will give us about 768 cardiovascular events during a mean exposure of 2 years. When we're done we hope to be able to advise both prescribing physicians and patients about the pain reliever you can take that carries the lowest risk. Now, there are also differential risks for gastrointestinal (GI) toxicity, so the primary endpoints are cardiovascular toxicity, but we're also going to find out about GI bleeding and GI tolerance by collecting ulcer events, and we're going to find out about arthritis efficacy. The COX2 inhibitors were very good pain relievers so if there is a relative risk for cardiovascular events we need to establish the relative benefit on symptomatic pain. We can then inform patients about what benefits they might expect and at what risk.

\section{How do you respond to criticism that the trial is unethical?}

If you don't know which of the three drugs is safest then there's not an ethical question, there is equipoise, which is what is required for a clinical trial. I feel very strongly that any of the three drugs we're studying could prove to be superior for safety, and if that's the case then the trial is entirely ethical.

What about suggestions that the trial will give Pfizer more time to protect Celebrex? This trial has a unique governance model - the design is not Pfizer's, it's ours. It's an academically directed trial designed to answer a scientific question, not a marketingdriven study by Pfizer. It also doesn't buy anybody any time, because all three of these drugs are already being prescribed in the dark by people who don't know their relative safety. What would be inappropriate is to do the kind of trials that have been done in the past that study smaller numbers of patients for shorter lengths of time and exclude patients at high cardiovascular risk. Every other trial has been designed to minimize the potential hazards of the drugs - we haven't done that.

\section{How did you end up getting involved with Pfizer in this study?}

I think Pfizer recognized that this trial had to provide a definitive answer and that it had to be done by people who would be trusted. I have been a very outspoken critic on issues of drug safety, and I believe that Pfizer asked me to direct this programme because it knew that the trial goes beyond answering conventional scientific questions; it's in the realm of the public and the media, and that means it has to be done right. One thing I insisted we do, and this is unprecedented in a pharma trial to my knowledge, is to put the entire trial database in the public domain housed at the US National Heart, Lung and Blood Institute one year after the trial is finished. The results have to pass the scrutiny of the most critical individuals and so we will do it in a way that's never been done before.

\section{Have there been any specific challenges to setting up this trial?}

We have a problem with the European Agency for the Evaluation of Medicinal Products (EMEA), which has declared celecoxib to be contraindicated in patients with cardiovascular disease, so we are not allowed to do the trial in EU countries. What's fascinating about this, and it's almost inexplicable, is that the EMEA has had input into the trial design. So they want to see the results, and they want input into the design, but they won't let us do the trial in their countries. For example, the EMEA requested that we allow rheumatoid arthritis patients in the trial, and we agreed to that in large part to give the EMEA the data that they seek - and yet they won't let us collect the data in the countries that they regulate. So we're going to do it in Eastern European countries, the US, Canada, Australia, South America and possibly in India, but we would have been a lot happier if the EMEA had granted us the necessary waivers to do the trial in EU countries.

\section{Was it difficult to avoid conflicts of interest?} Avoiding conflicts of interest is so pivotal in this trial that I asked each member of the executive committee, the governing group and the data safety and monitoring board to sign a statement that they will not accept any honoraria, consulting fees or any other monies, not only from Pfizer but from any maker of a drug in development or existing in this class. I think it was the right thing to do. We're really excited about this trial - and we're going to do this one right. 